Article

\title{
Quality Evaluation of Wild and Cultivated Schisandrae Chinensis Fructus Based on Simultaneous Determination of Multiple Bioactive Constituents Combined with Multivariate Statistical Analysis
}

\author{
Shuyu Chen ${ }^{1}{ }^{\circledR}$, Jingjing Shi ${ }^{1}$, Lisi Zou ${ }^{1}$, Xunhong Liu ${ }^{1, *}$, Renmao Tang ${ }^{2}$, Jimei Ma ${ }^{2}$, \\ Chengcheng Wang ${ }^{1}$, Mengxia Tan ${ }^{1}$ and Jiali Chen ${ }^{1}$ \\ 1 College of Pharmacy, Nanjing University of Chinese Medicine, Nanjing 210023, China; \\ 18305172513@163.com (S.C.); shijingjingquiet@163.com (J.S.); zlstcm@126.com (L.Z.); \\ ccw199192@163.com (C.W.); 18816250751@163.com (M.T.); 18994986833@163.com (J.C.) \\ 2 SZYY Group Pharmaceutical Limited, Taizhou 225500, China; ktang@vip.163.com (R.T.); \\ mjm0607@hotmail.com (J.M.) \\ * Correspondence: liuxunh1959@163.com; Tel./Fax: +86-25-8581-1524
}

Academic Editor: David Barker

Received: 7 March 2019; Accepted: 3 April 2019; Published: 4 April 2019

\begin{abstract}
Schisandrae Chinensis Fructus, also called wuweizi in China, was a widely used folk medicine in China, Korea, and Russia. Due to the limited natural resources and huge demand of wuweizi, people tend to cultivate wuweizi to protect this species. However, the quality of wild and cultivated herbs of the same species may change. Little attention has been paid to comparing wild and cultivated wuweizi based on simultaneous determination of its active components, such as lignans and organic acids. An analytical method based on UFLC-QTRAP-MS/MS was used for the simultaneous determination of 15 components, including 11 lignans (schisandrin, gomisin $\mathrm{D}$, gomisin $\mathrm{J}$, schisandrol B, angeloylgomisin $\mathrm{H}$, schizantherin B, schisanhenol, deoxyschizandrin, $\gamma$-schisandrin, schizandrin $\mathrm{C}$, and schisantherin) and 4 organic acids (quinic acid, D(-)-tartaric acid, L-(-)-malic acid, and protocatechuic acid) in wuweizi under different ecological environments. Principal components analysis (PCA), partial least squares discrimination analysis (PLS-DA), independent sample t-test, and gray relational analysis (GRA) have been applied to classify and evaluate samples from different ecological environments according to the content of 15 components. The results showed that the differential compounds (i.e., quinic acid, L-(-)-malic acid, protocatechuic acid, schisandrol B) were significantly related to the classification of wild and cultivated wuweizi. GRA results demonstrated that the quality of cultivated wuweizi was not as good as wild wuweizi. The protocol not just provided a new method for the comprehensive evaluation and quality control of wild and cultivated wuweizi, but paved the way to differentiate them at the chemistry level.
\end{abstract}

Keywords: Schisandrae Chinensis Fructus; wild; cultivated; multiple bioactive components; simultaneous quantitation

\section{Introduction}

Schisandrae Chinensis Fructus (wuweizi) is the dried ripe fruit of the magnolia plant Schisandra chinensis (Turcz.) Baillon. Wuweizi was a folk medicine in China, Korea, and Russia, which was used as a sedative and tonic. With the ability to replenish vital energy and promote fluid production, benefitting the kidneys and tranquilizing the mind, wuweizi was used to treat seminal emission, excessive sweating, diarrhea, insomnia, fatigue, and neurasthenia in clinics $[1,2]$. Existing research studies have shown that lignans with a dibenzocyclooctadiene skeleton and organic 
acids are major types of phytochemicals in wuweizi [3,4]. It has been demonstrated that lignans have abundant bioactivities, including antihepatotoxic, antioxidant, antitumor, nervous system protection, and anticancer properties [5-7]. Organic acids have the beneficial pharmacological effects of arresting coughs and removing phlegm $[2,8,9]$.

With the deeper understanding on the effectiveness of wuweizi, many industries, such as medicine, prescriptions, health products, and food and beverage, have a wide range of applications for this species. Various kinds of products, such as beverages, fermented wine, jam, tea, jelly, and dye, have aroused the demand for wuweizi. Due to excessive logging of unsustainable wild resources, the wild resources of wuweizi in northeast China have rapidly decreased and cannot meet the increasing demand of the markets. Therefore, planting cultivars has become a trend throughout northeast China. Unlike the wild ecological environment, the cultivated ecological environment is an artificial intervention. When plants face adversity, specialized metabolites accumulate significantly more than usual [10]. It is generally believed that most of the bioactive components isolated from herbs belong to specialised metabolites [11]. Varied ecological environments can lead to plants producing and accumulating various specialized metabolites, which cause uneven qualities of wuweizi. It is the amount or proportion among the medicinal constituents that mainly contribute to the quality difference for herbal medicine of the same species [12,13].

Nowadays, wuweizi is officially documented in Chinese, Japanese, European, Korean, and Russian pharmacopoeias, and other worldwide pharmacopoeial monographs [2]. Schisandrin is the quality indicator in the Chinese and United States pharmacopoeia. In addition, the content of lignans as the sum of the percentages of schisandrin, schisandrol B, deoxyschisandrin, and $\gamma$-schisandrin shall not be less than $0.95 \%$ by HPLC for quality control, as has been listed in the United States pharmacopeia. The control of several simple components may provide certain guidance, but for the multi-component and multi-target medicinal plants, there are still some limitations [14] because the pharmacological effect of wuweizi was usually a comprehensive effect of various kinds of compounds. For example, lignans and organic acids all have pharmacological effects, but they take different effects. However, most reports of quantitative analysis were focused on each particular class of compounds or certain active ingredients for the quality control of wuweizi [4,15-18]. The analysis of particular lignans alone was not sufficient for further study of the wuweizi quality control. Therefore, it is necessary to study the differences between wild and cultivated wuweizi and comprehensively evaluate the quality of them based on the simultaneous determination of lignans and organic acids.

The aim of this paper is to evaluate the quality of wild and cultivated wuweizi based on the simultaneous determination of multiple bioactive constituents combined with multivariate statistical analysis. A reliable method based on ultra-fast performance liquid chromatography coupled with triple quadrupole-linear ion trap mass spectrometry (UFLC-QTRAP-MS/MS) was used to simultaneously determine the content of 15 constituents, including 11 lignans and 4 organic acids in 12 batches of wuweizi samples from different ecosystems. Furthermore, principal component analysis (PCA) was introduced to get a good overview of the sample distribution. Partial least squares-discriminant analysis (PLS-DA) and t-tests were performed to show the difference of each compound between two types of wuweizi according to the contents of the tested constituents. Gray relational analysis (GRA) was carried out for the comprehensive evaluation. The protocol not just provided a new method for the comprehensive evaluation and quality control of wild and cultivated wuweizi, but allowed for differentiation of them at the chemistry level.

\section{Results and Discussion}

\subsection{Optimization of Extraction Conditions}

The orthogonal experiment was carried out to obtain a satisfactory extraction efficiency of major compounds in samples. Extraction solvent, material-solvent ratio, and ultrasonic time were optimized 
through 9 extraction experiments. The orthogonal table of levels and factors was shown in Table S1. The total peak area was calculated for evaluation of the orthogonal experiment, and the results were shown in Table S2. Finally, the optimum extraction conditions were achieved through ultrasonic extraction with a 40:1 ratio of methanol for $40 \mathrm{~min}$ at room temperature.

\subsection{Optimization of UFLC Conditions}

The peak area of schisandrin, theoretical plate number, resolution, and retention time were taken into consideration to investigate the different chromatographic columns, flow rates, column temperatures and mobile phases. Three kinds of columns, including an Agilent ZORBAX SB-C 18 column $(250 \mathrm{~mm} \times 4.6 \mathrm{~mm}, 5 \mu \mathrm{m})$, a XBridge $\mathrm{C}_{18}(100 \mathrm{~mm} \times 4.6 \mathrm{~mm}, 3.5 \mu \mathrm{m})$, and a Synergi ${ }^{\mathrm{TM}}$ Hydro-RP $100 \AA(100 \mathrm{~mm} \times 2.0 \mathrm{~mm}, 2.5 \mu \mathrm{m})$, were all compared to test samples. The results showed that the separation effect of three types of chromatographic columns were quite different, and the last one had the desirable resolution, peak shape, and retention time. Consequently, the column of Synergi ${ }^{\mathrm{TM}}$ Hydro-RP $100 \AA(100 \mathrm{~mm} \times 2.0 \mathrm{~mm}, 2.5 \mu \mathrm{m})$ was employed for this analysis. To achieve an efficient and rapid analysis, several UFLC parameters, including different kinds of mobile phases (methanol/water, acetonitrile/water, acetonitrile/0.1\% aqueous formic acid, acetonitrile containing $0.1 \%$ formic acid solution/water, and acetonitrile containing $0.1 \%$ formic acid solution/ $0.1 \%$ aqueous formic acid), flow rates $(0.3 \mathrm{~mL} / \mathrm{min}, 0.35 \mathrm{~mL} / \mathrm{min}$ and $0.4 \mathrm{~mL} / \mathrm{min})$, and column temperatures $(30,35$, and $40^{\circ} \mathrm{C}$ ) were examined systematically. When acetonitrile aqueous solution was used for gradient elution, the shape of the peaks were better. When the flow rate was $0.40 \mathrm{~mL} / \mathrm{min}$, the resolution was better. The results of resolution under three column temperatures were all satisfactory, however, the column temperature of $40^{\circ} \mathrm{C}$ had a higher theoretical plate number. Finally, it was determined that a gradient elution using water as eluent $\mathrm{A}$ and acetonitrile as eluent $\mathrm{B}$ at a flow rate of $0.4 \mathrm{~mL} / \mathrm{min}$ under the column temperature of $40{ }^{\circ} \mathrm{C}$ resulted in the desired separation in a short analysis time.

\subsection{Optimization of MS Conditions}

In order to develop a sensitive and accurate quantitative method, individual solutions of all standard compounds (about $100 \mathrm{ng} / \mathrm{mL}$ ) were examined separately with infusion mode by a full-scan mass spectrometry method in both positive and negative modes. After trial and error inspection, we found that lignans show maximum sensitivity under the positive ion mode. However, organic acids display desirable results in the negative ion mode. ESI, the electrospray ionizationsource of MS, could obtain abundant fragment ions of compounds. MRM (multiple reaction monitoring) is suitable for the quantification of components as a promising technology for the sensitivity and robustness. MRM transition from the MS/MS spectrum was chosen when the most abundant fragment ions appeared. For example, compounds 1, 2, 3, and 4 the showed most intense ion for $[\mathrm{M}-\mathrm{H}]^{-}$. All the optimum values, including retention time $\left(t_{R}\right)$, mass data $(m / z)$, precursor and product ions, fragmentor voltage (FV), and collision energy (CE) for each compound are summarized in Table 1. 
Table 1. Precursor/product ion pairs and parameters for MRM of the target compounds.

\begin{tabular}{|c|c|c|c|c|c|c|c|}
\hline No & Coumpounds & $t_{R}(\min )$ & $\begin{array}{c}\text { Mass Data } \\
(m / z)\end{array}$ & $\begin{array}{l}\text { Precursor } \\
\text { Ion }\end{array}$ & $\begin{array}{l}\text { Product } \\
\text { Ion }\end{array}$ & FV (V) & $\mathrm{CE}(\mathrm{eV})$ \\
\hline 1 & Quinic acid & 0.79 & 191.1[M - H]- & 191.1 & 85.02 & -120 & -26 \\
\hline 2 & $\mathrm{D}(-)$-Tartaric acid & 0.82 & 149.0[M - H]- & 149.0 & 87 & -55 & -16 \\
\hline 3 & L-(-)-Malic acid & 0.84 & $133.0[\mathrm{M}-\mathrm{H}]-$ & 133.0 & 114.9 & -80 & -14 \\
\hline 4 & Protocatechuic acid & 1.11 & $153.0[\mathrm{M}-\mathrm{H}]-$ & 153.0 & 106 & -85 & -16 \\
\hline 5 & Schisandrin & 5.65 & $433.3[\mathrm{M}+\mathrm{H}]^{+}$ & 433.3 & 415.34 & 146 & 13 \\
\hline 6 & Gomisin D & 5.75 & $553.3[\mathrm{M}+\mathrm{Na}]^{+}$ & 553.3 & 507.32 & 21 & 35 \\
\hline 7 & Gomisin J & 5.92 & $389.3[\mathrm{M}+\mathrm{H}]^{+}$ & 389.3 & 287.1 & 156 & 27 \\
\hline 8 & Schisandrol B & 6.11 & $417.3[\mathrm{M}+\mathrm{H}]^{+}$ & 417.3 & 399.2 & 131 & 15 \\
\hline 9 & Angeloylgomisin $\mathrm{H}$ & 6.67 & $501.3[\mathrm{M}+\mathrm{H}]^{+}$ & 501.3 & 401.2 & 146 & 11 \\
\hline 10 & Schizantherin B & 7.34 & $515.3[\mathrm{M}+\mathrm{H}]^{+}$ & 515.3 & 415.2 & 56 & 11 \\
\hline 11 & Schisanhenol & 7.63 & $403.2[\mathrm{M}+\mathrm{H}]^{+}$ & 403.2 & 340.2 & 1 & 27 \\
\hline 12 & Deoxyschizandrin & 8.83 & $417.3[\mathrm{M}+\mathrm{H}]^{+}$ & 417.3 & 316.18 & 241 & 31 \\
\hline 13 & $\gamma$-schisandrin & 9.50 & $401.3[\mathrm{M}+\mathrm{H}]^{+}$ & 401.3 & 300.15 & 231 & 31 \\
\hline 14 & Schizandrin C & 9.85 & $385.2[\mathrm{M}+\mathrm{H}]^{+}$ & 385.2 & 285.16 & 201 & 29 \\
\hline 15 & Schisantherin & 11.96 & $537.4[\mathrm{M}+\mathrm{H}]^{+}$ & 537.4 & 282.3 & 56 & 15 \\
\hline
\end{tabular}

\subsection{UFLC Method Validation}

All method validations of quantification were performed using the UFLC-QTRAP-MS/MS technique. Each standard calibration curve was constructed by plotting the peak areas $(\mathrm{Y})$ against the corresponding concentrations $(X)$, which achieved appropriate determination coefficients $\left(r^{2}>0.9991\right)$, and the test range covered the concentrations of investigated compounds in samples. The limits of detection (LOD) and limits of quantification (LOQ) were in the ranges of $0.49-2.95 \mathrm{ng} / \mathrm{mL}$ and $1.38-12.65 \mathrm{ng} / \mathrm{mL}$ of 15 analytes, respectively. The relative standard deviation (RSD) values of intra-day and inter-day variations of 15 components ranged from $0.67 \%$ to $3.09 \%$ and $0.15 \%$ to $2.63 \%$, respectively. The RSD of repeatability and stability tests of the 15 analytes were less than $5 \%$, and the overall recoveries varied between $95.62 \%$ and $99.97 \%$, with RSDs less than $4.12 \%$, demonstrating that this method was validated for all kinds of analytes. The detailed results of each method validation were presented in Table 2 and Table S3. 
Table 2. Regression equation, limits of detection, limits of quantification, precision, repeatability, stability, and recovery of 30 investigated compounds.

\begin{tabular}{|c|c|c|c|c|c|c|c|c|c|c|c|c|c|c|c|c|}
\hline \multirow{3}{*}{ No. } & \multirow{3}{*}{ Compounds } & \multirow{3}{*}{$\begin{array}{c}\text { Regression } \\
\text { Equation }\end{array}$} & \multirow{3}{*}{$r^{2}$} & \multirow{3}{*}{$\begin{array}{c}\text { Liner Range } \\
(\mathrm{ng} / \mathrm{mL})\end{array}$} & \multirow{3}{*}{$\begin{array}{l}\text { LODs } \\
\text { (ng/mL) }\end{array}$} & \multirow{3}{*}{$\begin{array}{l}\mathrm{LOQs} \\
\text { (ng/mL) }\end{array}$} & \multicolumn{2}{|c|}{$\begin{array}{c}\text { Precision (RSD, } \\
\% \text { ) }\end{array}$} & \multirow{3}{*}{$\begin{array}{c}\text { Repeatability } \\
(\text { RSD, \%) }(n=6)\end{array}$} & \multirow{3}{*}{$\begin{array}{c}\text { Stability (RSD, \%) } \\
(n=6)\end{array}$} & \multicolumn{6}{|c|}{ Recovery $(\%)(n=3)$} \\
\hline & & & & & & & \multirow{2}{*}{\multicolumn{2}{|c|}{$\begin{array}{l}\text { Intra-Day Inter-Day } \\
(n=6) \quad(n=3)\end{array}$}} & & & \multicolumn{2}{|c|}{ Low } & \multicolumn{2}{|c|}{ Medium } & \multicolumn{2}{|c|}{ High } \\
\hline & & & & & & & & & & & Mean & RSD & Mean & RSD & Mean & RSD \\
\hline 1 & Quinic acid & $Y=1330 X+76100$ & 1.0000 & $133-13300$ & 2.66 & 6.57 & 2.40 & 2.63 & 3.5 & 1.1 & 99.55 & 0.22 & 99.65 & 0.36 & 99.83 & 0.05 \\
\hline 2 & $\mathrm{D}(-)$-Tartaric acid & $Y=1240 X+1190$ & 0.9995 & $3.75-24.9$ & 0.49 & 1.62 & 3.09 & 1.60 & 2.1 & 2.3 & 95.62 & 2.1 & 98.07 & 0.57 & 97.34 & 0.74 \\
\hline 3 & L-(-)-Malic acid & $Y=2040 X+149000$ & 0.9991 & $148-29500$ & 2.95 & 12.42 & 1.79 & 1.45 & 3.2 & 1.2 & 99.92 & 0.06 & 99.93 & 0.03 & 99.96 & 0.06 \\
\hline 4 & Protocatechuic acid & $\mathrm{Y}=162 \mathrm{X}+3100$ & 0.9997 & $255-12800$ & 1.96 & 9.03 & 2.31 & 127 & 25 & 33 & 9998 & 0.01 & 9996 & 0.02 & 9993 & 0.06 \\
\hline 5 & Schisandrin & $\mathrm{Y}=21.3 \mathrm{X}+1550$ & 0.9991 & $116-9320$ & 136 & 784 & 306 & 186 & 07 & 31 & 9936 & 03 & 998 & 01 & 9963 & 0.06 \\
\hline 6 & Gomisin D & $\mathrm{Y}=3770 \mathrm{X}+34900$ & 09992 & $101-506$ & 084 & 661 & 111 & 081 & 15 & 12 & 96.63 & 236 & 9696 & 187 & 96.33 & 4.12 \\
\hline 7 & Gomisin I & $\mathrm{Y}=739 \mathrm{X}+7100$ & 09993 & $233-931$ & 153 & 607 & 203 & 126 & 30 & 30 & 98.63 & 062 & 98.59 & 0.54 & 977 & 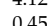 \\
\hline 8 & Schisandrol B & $Y=4140 X+260000$ & 0.9997 & $107-5350$ & $\begin{array}{l}1.05 \\
1.92\end{array}$ & 8.15 & 2.056 & 0.31 & 24 & 0.0 & (2) & 0.028 & $\begin{array}{l}90.035 \\
9085\end{array}$ & 0.078 & 9913 & 0.79 \\
\hline 9 & Angeloyloomisin $\mathrm{H}$ & $Y=25800 X+623000$ & 0.9991 & $565-2260$ & 1.92 & 538 & 245 & 0.015 & 16 & 31 & 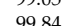 & 0.00 & 90.05 & 0. & 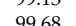 & 032 \\
\hline 10 & Schizantherin B & $Y=240 X+3640$ & 0.9998 & $\begin{array}{l}5.02-2200 \\
552-2210\end{array}$ & 1.00 & 12.00 & 100 & 0.54 & 1.0 & $w_{3}+1$ & 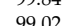 & 0.24 & 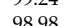 & 0.73 & 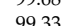 & 0.59 \\
\hline 11 & $\begin{array}{l}\text { Schisanhenol } \\
\text { Sol }\end{array}$ & $\mathrm{Y}=2900 \mathrm{X}+10800$ & 0.9999 & $199-1194$ & 1.99 & 5.82 & $\begin{array}{l}1.00 \\
2.59\end{array}$ & 1.58 & 4.9 & 1.5 & 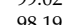 & 1. 107 & $\begin{array}{l}30.96 \\
9863\end{array}$ & 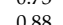 & 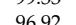 & 3.03 \\
\hline 12 & Deoxyschizandrin & $\mathrm{Y}=26600 \mathrm{X}+217000$ & 1.0000 & $24-2400$ & 2.91 & $\begin{array}{r}0.02 \\
11.21\end{array}$ & 0.87 & $\begin{array}{l}1.1 .10 \\
1.10\end{array}$ & 1.6 & 1.3 & 97.83 & 0.74 & $\begin{array}{l}90.00 \\
98.86\end{array}$ & 0.34 & 99.42 & 0.02 \\
\hline 13 & $\gamma$-schisandrin & $\mathrm{Y}=15700 \mathrm{X}+214000$ & 1.0000 & $15.2-4560$ & 1.79 & 6.92 & 1.91 & 0.43 & $\begin{array}{l}1.0 \\
0.5\end{array}$ & 1.1 & 99.63 & 0.3 & $\begin{array}{l}90.00 \\
99.75\end{array}$ & 0.09 & 99.69 & 0.13 \\
\hline 14 & Schizandrin C & $Y=6410 X+24300$ & 0 & 293-1470 & $055^{2}$ & 138 & 205 & 0.66 & 38 & 1.1 & 9857 & 041 & 9964 & 0.01 & 9824 & 0.13 \\
\hline 15 & $\begin{array}{l}\text { Schisantherin } \\
\text { Sols }\end{array}$ & $\mathrm{Y}=25 \mathrm{X}+1860$ & 09998 & $12-7200$ & 159 & 562 & 0.67 & $\begin{array}{l}0.00 \\
1.61\end{array}$ & 2.0 & 10 & 9989 & $\begin{array}{l}0.11 \\
0.04\end{array}$ & $\begin{array}{l}71.00 \\
9989\end{array}$ & 0.04 & $\begin{array}{l}30.24 \\
99.97\end{array}$ & 0.02 \\
\hline
\end{tabular}




\subsection{Quantification of Lignans and Organic Acids}

Twelve batches of cultivated and wild wuweizi were collected from Heilongjiang, Jilin, and Liaoning and dealt with using the same processing method (sun drying). Sample information is listed in Table 3. The validated analytical method of UFLC-QTRAP-MS/MS was successfully applied to the simultaneous determination of 11 lignans and 4 organic acids in wuweizi. Each sample was determined three times and the quantitative results of 15 compounds are presented in Table 4 . Total ion chromatograms of the representative wild and cultivated samples are shown in Figure S1. Typical MRM chromatograms are shown in Figure 1. The histogram (Figure 2) suggests lignans were found in higher concentrations in wild than cultivated-type. However, the content of organic acids was slightly higher in the cultivated-type. It was clearly shown that the total contents of 15 compounds varied from $25,598.77 \mu \mathrm{g} / \mathrm{g}$ to $35,179.73 \mu \mathrm{g} / \mathrm{g}$. The total contents of each type of constituent was also calculated, and 11 lignans ranged from $14,960.30 \mu \mathrm{g} / \mathrm{g}$ to $22,853.62 \mu \mathrm{g} / \mathrm{g}$, and in the following order: (highest) S1 (wild) $>$ S6 (wild) $>\mathrm{S} 3$ (wild) $>\mathrm{S} 5$ (wild) $>\mathrm{S} 4$ (wild) $>\mathrm{S} 10$ (cultivated) $>\mathrm{S} 2$ (wild) $>$ S8 (cultivated) $>$ S7 (cultivated) $>$ S11 (cultivated) $>$ S9 (cultivated) $>$ S12 (cultivated) (lowest). The four organic acids ranged from $9075.64 \mu \mathrm{g} / \mathrm{g}$ to $13,646.81 \mu \mathrm{g} / \mathrm{g}$. By comparing the amounts, it was found that the compounds of wuweizi from different ecosystems were quite different. In this study, the contents of lignans were similar to previous studies [4,15-18]. However, the contents of organic acids were slightly lower than the reported, which may be related to the harvest period, processing, and origin [19].

Table 3. Detailed information of samples.

\begin{tabular}{|c|c|c|c|c|}
\hline Sample No. & Habitats & GPS Records & Harvesting Time & $\begin{array}{c}\text { Processing } \\
\text { Method }\end{array}$ \\
\hline S1 & Mulan, Heilongjiang & $\begin{array}{l}45^{\circ} 56^{\prime} 54^{\prime \prime} \mathrm{N}, \\
128^{\circ} 02^{\prime} 14^{\prime \prime} \mathrm{E}\end{array}$ & 10 August 2017 & sun drying \\
\hline S2 & Jingyu, Jilin & $\begin{array}{l}42^{\circ} 23^{\prime} 11^{\prime \prime} \mathrm{N} \\
126^{\circ} 48^{\prime} 28^{\prime \prime} \mathrm{E}\end{array}$ & 14 August 2017 & sun drying \\
\hline S3 & Jingyu, Jilin & $\begin{array}{l}42^{\circ} 23^{\prime} 11^{\prime \prime} \mathrm{N}, \\
126^{\circ} 48^{\prime} 28^{\prime \prime} \mathrm{E}\end{array}$ & 17 August 2017 & sun drying \\
\hline $\mathrm{S} 4$ & Xinbin, Liaoning & $\begin{array}{l}41^{\circ} 43^{\prime} 53^{\prime \prime} \mathrm{N} \\
125^{\circ} 02^{\prime} 01^{\prime \prime} \mathrm{E}\end{array}$ & 14 August 2017 & sun drying \\
\hline S5 & Hengren, Liaoning & $\begin{array}{l}41^{\circ} 15^{\prime} 13^{\prime \prime} \mathrm{N} \\
125^{\circ} 22^{\prime} 15^{\prime \prime} \mathrm{E}\end{array}$ & 10 August 2017 & sun drying \\
\hline S6 & Baoqing, Heilongjiang & $\begin{array}{l}46^{\circ} 19^{\prime} 29^{\prime \prime} \mathrm{N} \\
132^{\circ} 11^{\prime} 22^{\prime \prime} \mathrm{E}\end{array}$ & 20 August 2017 & sun drying \\
\hline S7 & Jingyu, Jilin & $\begin{array}{l}42^{\circ} 23^{\prime} 11^{\prime \prime} \mathrm{N}, \\
126^{\circ} 48^{\prime} 28^{\prime \prime} \mathrm{E}\end{array}$ & 16 August 2017 & sun drying \\
\hline S8 & Jingyu, Jilin & $\begin{array}{l}42^{\circ} 23^{\prime} 11^{\prime \prime} \mathrm{N} \\
126^{\circ} 48^{\prime} 28^{\prime \prime} \mathrm{E}\end{array}$ & 17 August 2017 & sun drying \\
\hline S9 & Jingyu, Jilin & $\begin{array}{l}42^{\circ} 23^{\prime} 11^{\prime \prime} \mathrm{N}, \\
126^{\circ} 48^{\prime} 28^{\prime \prime} \mathrm{E}\end{array}$ & 20 August 2017 & sun drying \\
\hline $\mathrm{S} 10$ & Fengcheng, Liaoning & $\begin{array}{l}41^{\circ} 48^{\prime} 19^{\prime \prime} \mathrm{N} \\
123^{\circ} 27^{\prime} 47^{\prime \prime} \mathrm{E}\end{array}$ & 10 August 2017 & sun drying \\
\hline S11 & Shuangyang, Jilin & $\begin{array}{l}43^{\circ} 31^{\prime} 22^{\prime \prime} \mathrm{N}, \\
125^{\circ} 39^{\prime} 31^{\prime \prime} \mathrm{E}\end{array}$ & 10 August 2017 & sun drying \\
\hline $\mathrm{S} 12$ & Heihe, Heilongjiang & $\begin{array}{l}50^{\circ} 14^{\prime} 37^{\prime \prime} \mathrm{N}, \\
127^{\circ} 31^{\prime} 16^{\prime \prime} \mathrm{E}\end{array}$ & 16 August 2017 & sun drying \\
\hline
\end{tabular}


Table 4. Contents of 15 compounds in wuweizi ( $\mu \mathrm{g} / \mathrm{g}$, mean $\pm \mathrm{SD}, n=3)$.

\begin{tabular}{|c|c|c|c|c|c|c|c|c|c|c|c|c|}
\hline \multirow{2}{*}{ Analyte } & \multicolumn{6}{|c|}{ Wild } & \multicolumn{6}{|c|}{ Cultivated } \\
\hline & $\mathrm{S} 1^{\mathrm{a}}$ & $\mathrm{S}^{\mathrm{a}}$ & $\mathrm{S}^{\mathrm{a}}{ }^{\mathrm{a}}$ & $54^{\mathrm{a}}$ & $\mathrm{S} 5^{\mathrm{a}}$ & $56^{a}$ & $\mathrm{~S}^{\mathrm{a}}{ }^{\mathrm{a}}$ & $58^{a}$ & $\mathrm{S9} \mathrm{a}^{\mathrm{a}}$ & $\mathrm{S} 10^{\mathrm{a}}$ & $\mathrm{S} 11^{\mathrm{a}}$ & $\mathrm{S} 12^{\mathrm{a}}$ \\
\hline $1^{\mathrm{b}}$ & $5093.16 \pm 30.08$ & $4213.46 \pm 7.52$ & $4283.63 \pm 18.92$ & $5155.81 \pm 4.34$ & $3461.58 \pm 52.63$ & $4070.60 \pm 22.56$ & $5108.20 \pm 15.04$ & $5243.53 \pm 30.08$ & $5228.50 \pm 22.56$ & $6220.98 \pm 15.04$ & $6175.86 \pm 22.56$ & $6115.71 \pm 0.00$ \\
\hline $2^{\mathrm{b}}$ & $5.27 \pm 0.04$ & $6.23 \pm 0.01$ & $6.45 \pm 0.04$ & $6.80 \pm 0.03$ & $5.05 \pm 0.01$ & $4.73 \pm 0.01$ & $8.15 \pm 0.48$ & $8.31 \pm 0.32$ & $4.60 \pm 0.06$ & $7.06 \pm 0.01$ & $6.69 \pm 0.03$ & $8.31 \pm 0.32$ \\
\hline $3^{\mathrm{b}}$ & $657.03 \pm 0.57$ & $2050.65 \pm 1.98$ & $2687.42 \pm 1.13$ & $1564.71 \pm 0.85$ & $1245.42 \pm 0.28$ & $3781.21 \pm 2.26$ & $4196.57 \pm 8.49$ & $5413.89 \pm 5.66$ & $3585.78 \pm 5.09$ & $5379.58 \pm 19.81$ & $4673.53 \pm 2.55$ & $4884.48 \pm 11.32$ \\
\hline $4^{\mathrm{b}}$ & $3382.10 \pm 24.69$ & $5061.52 \pm 0.71$ & $3680.04 \pm 2.85$ & $5562.96 \pm 3.21$ & $4363.58 \pm 0.00$ & $4469.14 \pm 1.07$ & $2470.58 \pm 7.13$ & $2981.07 \pm 0.36$ & $1426.13 \pm 1.43$ & $1716.67 \pm 2.14$ & $2408.23 \pm 2.49$ & $2290.53 \pm 1.78$ \\
\hline $5^{\mathrm{b}}$ & $6171.36 \pm 46.95$ & $5847.42 \pm 72.88$ & $7110.33 \pm 37.56$ & $7287.17 \pm 9.77$ & $6457.75 \pm 4.69$ & $7482.79 \pm 5.42$ & $5847.42 \pm 54.14$ & $6451.49 \pm 2.71$ & $5093.11 \pm 49.31$ & $5988.26 \pm 101.02$ & $5847.42 \pm 16.93$ & $5510.95 \pm 42.34$ \\
\hline $6^{\mathrm{b}}$ & $107.63 \pm 0.31$ & $64.04 \pm 0.15$ & $61.48 \pm 1.6$ & $94.81 \pm 0.67$ & $93.75 \pm 1.62$ & $119.30 \pm 0.93$ & $37.16 \pm 0.46$ & $63.69 \pm 0.80$ & $42.29 \pm 0.77$ & $54.58 \pm 0.31$ & $60.86 \pm 0.41$ & $54.49 \pm 0.55$ \\
\hline $7^{\mathrm{b}}$ & $662.92 \pm 2.34$ & $601.58 \pm 0.78$ & $562.34 \pm 6.1$ & $389.13 \pm 2.07$ & $539.33 \pm 4.35$ & $617.82 \pm 2.82$ & $312.90 \pm 3.91$ & $309.29 \pm 3.13$ & $260.13 \pm 1.56$ & $478.89 \pm 4.06$ & $600.68 \pm 3.58$ & $382.81 \pm 1.35$ \\
\hline $8^{\mathrm{b}}$ & $4800.32 \pm 27.89$ & $2537.84 \pm 13.95$ & $3528.18 \pm 13.95$ & $2859.90 \pm 48.31$ & $3560.39 \pm 53.14$ & $3850.24 \pm 48.31$ & $1512.88 \pm 1.39$ & $1762.48 \pm 6.08$ & $1802.74 \pm 2.79$ & $2029.79 \pm 3.69$ & $3278.58 \pm 27.89$ & $3061.19 \pm 60.79$ \\
\hline $9^{\mathrm{b}}$ & $1814.35 \pm 5.92$ & $1216.16 \pm 38.76$ & $1518.49 \pm 3.88$ & $1344.07 \pm 11.63$ & $1446.14 \pm 8.07$ & $1690.97 \pm 22.35$ & $934.51 \pm 13.61$ & $916.42 \pm 4.48$ & $878.95 \pm 15.50$ & $1014.61 \pm 7.75$ & $1182.83 \pm 2.05$ & $1147.69 \pm 2.24$ \\
\hline $10^{\mathrm{b}}$ & $840.39 \pm 2.41$ & $547.33 \pm 4.17$ & $877.89 \pm 10.49$ & $684.83 \pm 15.02$ & $870.94 \pm 18.79$ & $714.00 \pm 12.50$ & $714.00 \pm 12.50$ & $557.06 \pm 6.36$ & $458.44 \pm 18.79$ & $726.50 \pm 8.33$ & $690.39 \pm 12.73$ & $1205.67 \pm 36.32$ \\
\hline $11^{\mathrm{b}}$ & $626.16 \pm 5.27$ & $528.46 \pm 10.53$ & $523.86 \pm 6.90$ & $485.93 \pm 10.34$ & $468.69 \pm 3.45$ & $508.92 \pm 12.11$ & $457.20 \pm 8.68$ & $481.33 \pm 1.99$ & $268.92 \pm 0.80$ & $341.1 \pm 0.00$ & $263.63 \pm 0.53$ & $245.59 \pm 0.34$ \\
\hline $12^{\mathrm{b}}$ & $1749.99 \pm 2.17$ & $978.71 \pm 4.34$ & $974.32 \pm 2.56$ & $965.35 \pm 3.76$ & $849.74 \pm 10.85$ & $971.36 \pm 41.41$ & $916.18 \pm 5.74$ & $1028.87 \pm 1.30$ & $602.78 \pm 4.24$ & $729.21 \pm 3.39$ & $538.68 \pm 4.7$ & $521.74 \pm 3.57$ \\
\hline $13^{\mathrm{b}}$ & $4046.88 \pm 3.18$ & $3149.85 \pm 3.68$ & $3165.77 \pm 1.84$ & $3158.56 \pm 0.37$ & $3504.42 \pm 9.73$ & $3774.06 \pm 16.03$ & $2635.63 \pm 0.74$ & $2647.94 \pm 5.15$ & $2208.87 \pm 5.74$ & $2495.71 \pm 6.05$ & $2489.55 \pm 6.37$ & $2374.27 \pm 5.44$ \\
\hline $14^{\mathrm{b}}$ & $787.58 \pm 1.00$ & $333.70 \pm 0.90$ & $689.40 \pm 2.38$ & $516.23 \pm 3.25$ & $704.79 \pm 2.05$ & $737.24 \pm 5.40$ & $249.41 \pm 0.95$ & $281.70 \pm 1.56$ & $247.38 \pm 0.00$ & $277.80 \pm 0.78$ & $437.55 \pm 1.58$ & $455.91 \pm 5.01$ \\
\hline $15^{\mathrm{b}}$ & $705.60 \pm 12.00$ & $708.27 \pm 2.31$ & $1692.27 \pm 2.31$ & - & $2046.93 \pm 8.33$ & $2386.93 \pm 6.11$ & $1889.60 \pm 4.00$ & $1421.60 \pm 0.00$ & $3490.93 \pm 4.62$ & $3093.60 \pm 6.93$ & $19.87 \pm 1.01$ & - \\
\hline Total & $31,450.74 \pm 55.65$ & $27,844.58 \pm 40.31$ & $31,361.47 \pm 100.10$ & $30,076.44 \pm 28.88$ & $29,619 \pm 105.38$ & $35,179.73 \pm 61.85$ & $27,289.59 \pm 94.08$ & $29,568.49 \pm 19.79$ & $25,598.77 \pm 42.20$ & $30,553.57 \pm 169.43$ & $28,674.25 \pm 21.71$ & $28,259.65 \pm 12.66$ \\
\hline
\end{tabular}

${ }^{\mathrm{a}}$ The sample number is same as in Table 3 ; $^{\mathrm{b}}$ the analyte number is the same as in Table 2. 


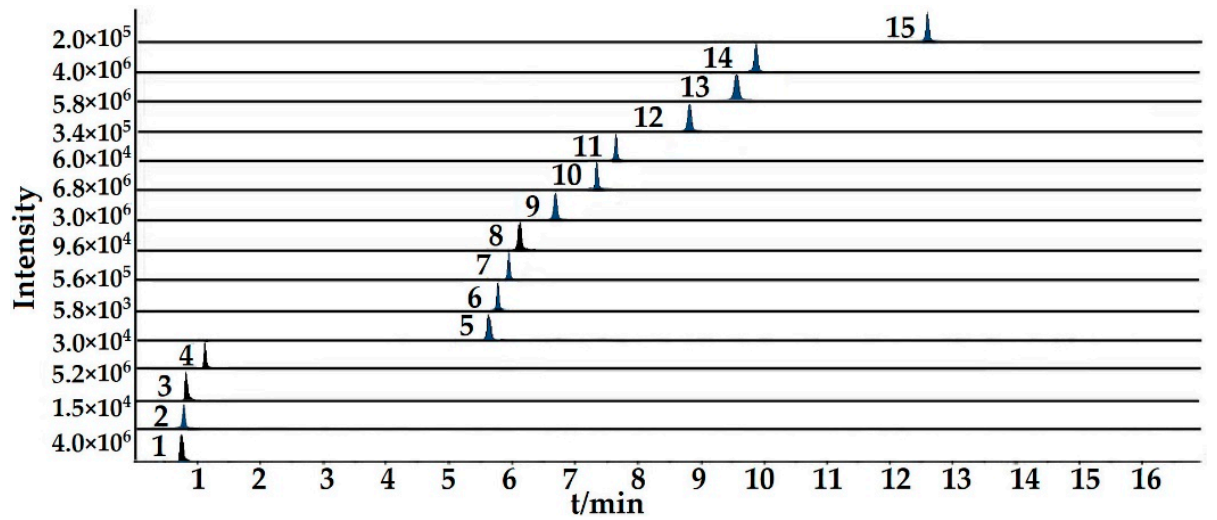

Figure 1. Representative extract ion chromatograms (XIC) of MRM chromatograms of 15 investigated compounds.

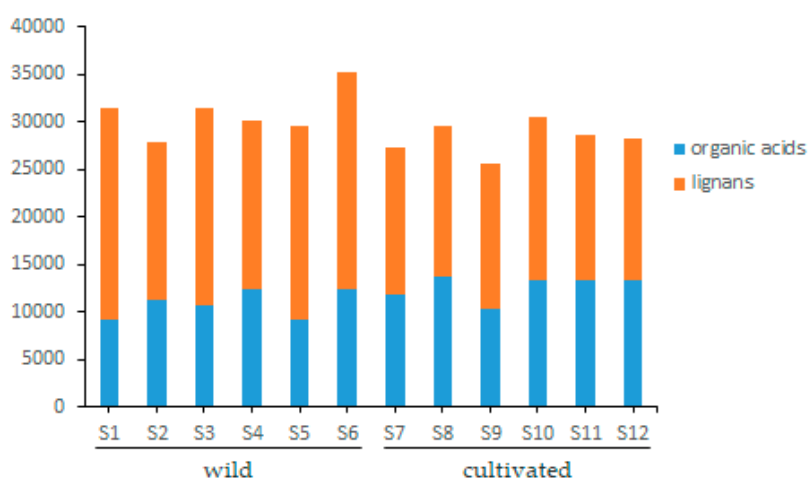

Figure 2. Histogram of the mean accumulative contents of different types of wuweizi from 12 batches.

\subsection{PCA of the Samples}

To evaluate the differences of components in wuweizi of two types, unsupervised PCA was performed. 12 Samples were set as observations, while the contents of 15 compounds were set as variables. The score scatter plot and the loading plot were displayed in Figure 3. In Figure 3, all samples were separated into two relative clusters, i.e., wild and cultivated wuweizi. This classification indicated that the content and distribution of chemical constituents varied between different types of wuweizi. The first and second principal components described $59.4 \%$ and $17.6 \%$ of the variability in the original observations, respectively, and the first two principal components accounted for $77.0 \%$ of the total variance.

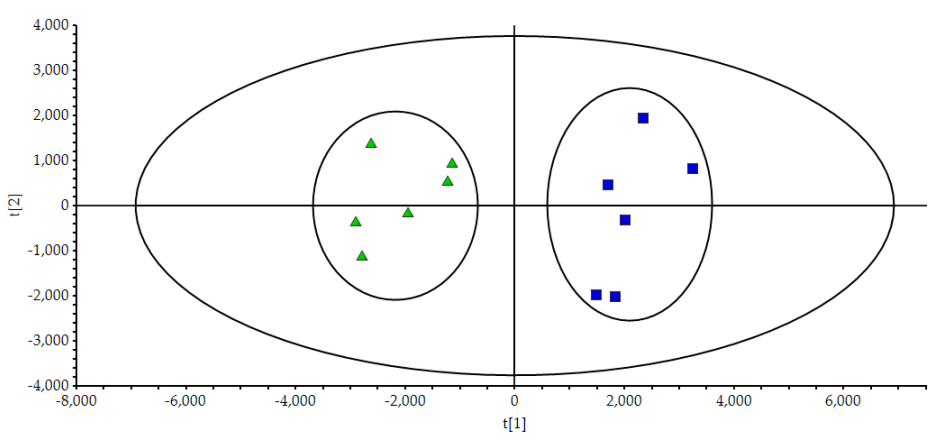

Figure 3. Score scatter plot of PCA processed data acquired from wild and cultivated wuweizi scanned by both positive and negative ion modes. Each of the green triangles represent a batch of wild wuweizi, while the blue squares represent a batch of cultivated ones. 


\subsection{PLS-DA of the Samples}

The selected initial data was further processed by PLS-DA in order to reveal differences in the chemical composition among wild and cultivated wuweizi. The results were shown in Figure 4. Model parameters were set as follows: confidence level was $95 \%, R^{2} Y=0.967$, and $Q^{2}=0.924$, and the parameters showed that the established PLS-DA model was effective. The PLS-DA score plot displayed that the two clusters representing the wild and cultivated groups were well separated, thereby indicating the remarkable differences between the two types.
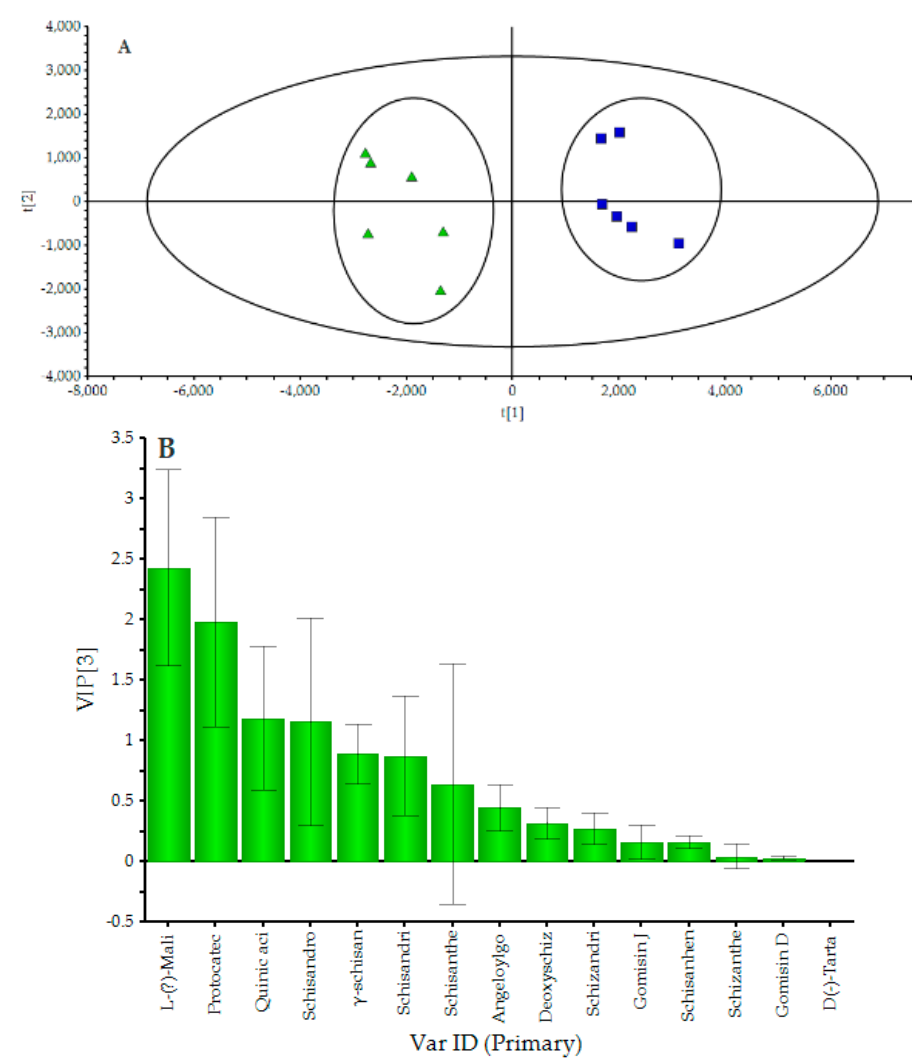

Figure 4. Score scatter plot (A) and VIP (B) by PLS-DA processed data obtained from wild and cultivated wuweizi scanned by positive and negative ion mode.

The VIP (variable importance for the projection) plot summarizes the importance of the variables both to explain $\mathrm{X}$ and to correlate to Y. The VIP plot is arranged from high to low, and the VIP-values greater than 1 indicated important variables, and four potential biological markers of quinic acid, L-(-)-malic acid, protocatechuic acid, and schisandrol B have high contributions to classification.

\subsection{T-test}

More than two-thirds of bioactive components quantified in this study showed a significant difference between two types of wuweizi, according to the T-test (Figure 5). Quantitation of major compounds, including protocatechuic acid, gomisin $\mathrm{D}$, angeloylgomisin $\mathrm{H}$, schisanhenol, and $\gamma$-schisandrin, showed strikingly higher levels $(p<0.01)$ in wild-type, while quinic acid and L-( -$)$-malic acid showed lower contents $(p<0.01)$ compared with cultivated-type. Secondly, quantitation of schisandrin, gomisin J, schisandrol B, and schizandrin C displayed highly contents $(p<0.05)$ in wild wuweizi than its cultivated-type. 

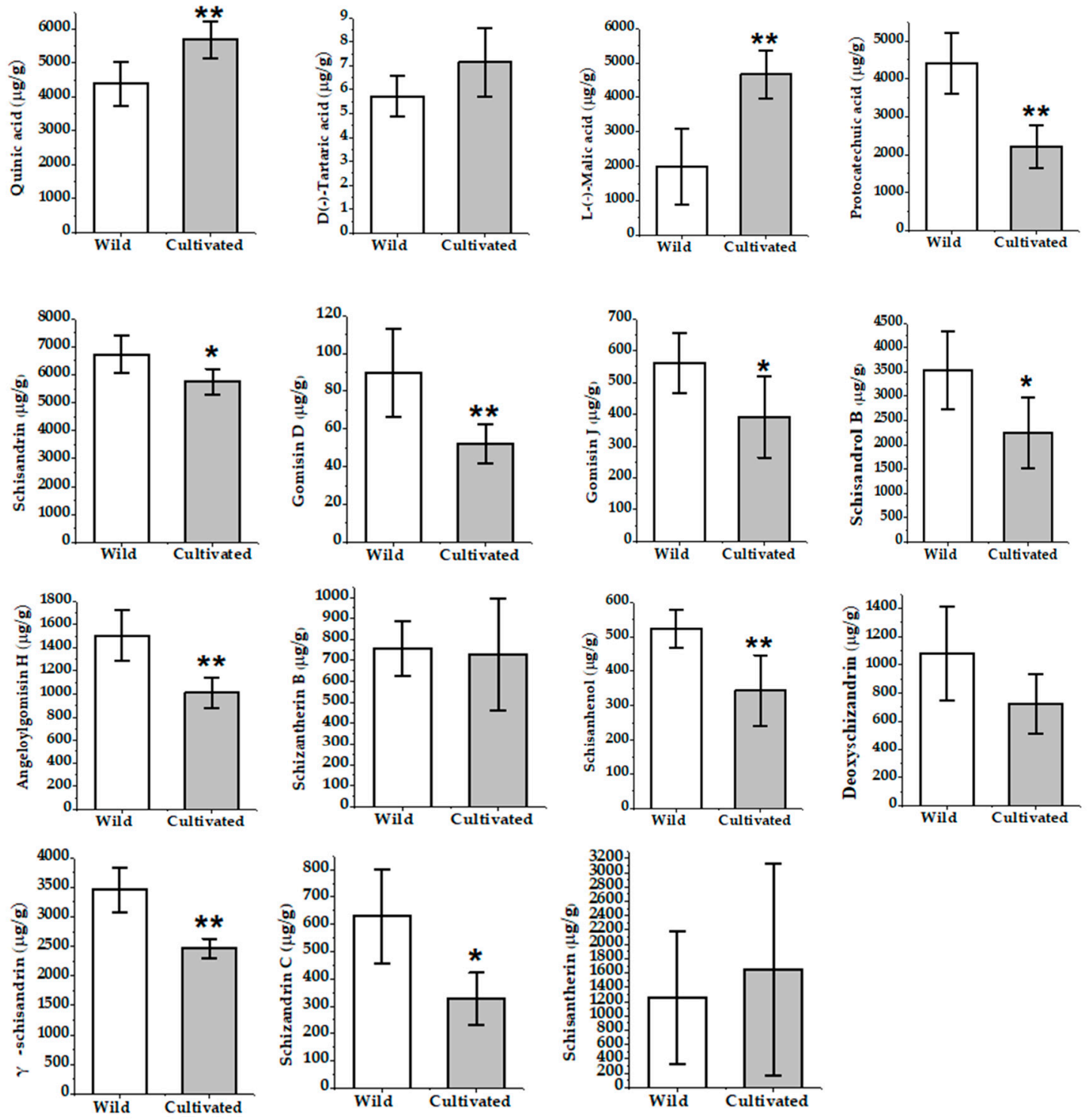

Figure 5. The contents of 15 compounds in wild and cultivated wuweizi $\left({ }^{*} p<0.05\right.$; $\left.{ }^{* *} p<0.01\right)$.

\subsection{Gray Relational Analysis (GRA)}

Because the contents of the 15 target components of lignans and organic acids in samples were different, it is difficult to judge the quality of samples intuitively. Therefore, gray relational analysis was carried out for comprehensive evaluation. It could be seen that the quality of wild wuweizi was better according to the results shown in Table S4. The quality sequencing of the samples was in the following order: S1 (wild) > S6 (wild) $>$ S3 (wild) $>$ S4 (wild) $>$ S5 (wild) $>$ S2 (wild) $>$ S10 (cultivated) $>\mathrm{S} 12$ (cultivated) $>\mathrm{S} 8$ (cultivated) $>\mathrm{S} 11$ (cultivated) $>\mathrm{S} 7$ (cultivated) $>$ S9 (cultivated).

\section{Materials and Methods}

\subsection{Plant Materials}

Twelve batches of cultivated and wild wuweizi dealing with the same processing method were studied in this research. The botanical origin of materials was authenticated by Prof. Xunhong Liu of the Nanjing University of Chinese Medicine. Voucher specimens were deposited at Herbarium in Nanjing University of Chinese Medicine. These samples were collected from Heilongjiang, Jilin, and Liaoning at around August 2017, dealing with sun drying for about 20 days. All batches of 
wuweizi were ripe when they were collected. Wild wuweizi grew under forests, valleys, and besides streams, places which were shady and moist. Cultivated wuweizi grew in arable land, which had enough sunshine and wind, or low-lying and rainy land. Detailed information is shown in Table 3.

\subsection{Chemicals and Reagents}

The reference compounds of L-(-)-malic acid (3), deoxyschizandrin (12), $\gamma$-schisandrin (13), schisandrin (5), schisantherin (15) were purchased from National Institutes for Food and Drug Control (Beijing, China). Quinic acid (1), D(-)-Tartaric acid (2), gomisin D (6), gomisin J (7), schizandrin C (14), schisandrol B (8), angeloylgomisin H (9), schizantherin B (10), and schisanhenol (11) were purchased from Nanjing Liangwei biotechnology Co., Ltd. (Nanjing, China). Protocatechuic acid (4) was purchased from Nanjing Spring and Autumn Biological Engineering Co., Ltd., China. The purity of all compounds by HPLC analysis was greater than $98 \%$. The structures of the 15 reference compounds are shown in Figure 6. Formic acid of MS grade, acetonitrile, and methanol of HPLC grade were purchased from Merck (Darmstadt, Germany). Ultrapure water was prepared using a Milli-Q water purification system (Millipore, Bedford, MA, USA).

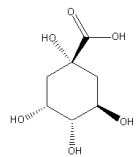

Quinic acid (1)
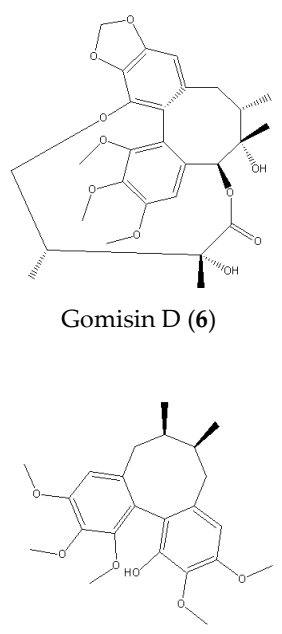

Schisanhenol (11)

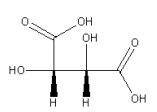

D(-)-Tartaric acid (2)

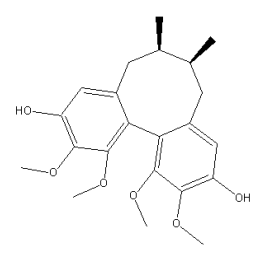

Gomisin J (7)

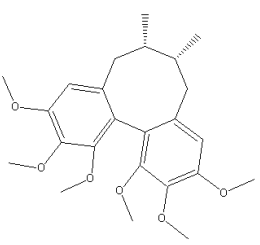

Deoxyschizandrin (12)

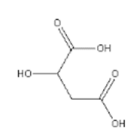

L-(-)-Malic acid (3)

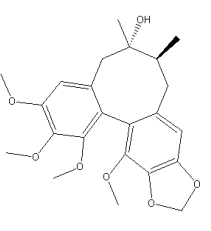

Schisandrol B (8)

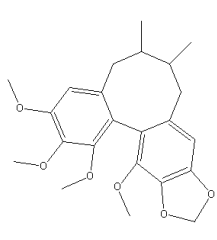

$\gamma$-schisandrin (13)
Protocatechuic acid (4)
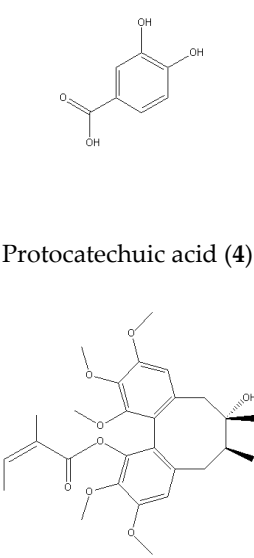

Angeloylgomisin H (9)

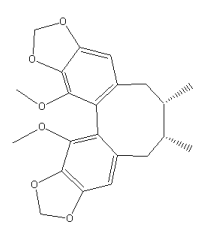

Schizandrin C (14)

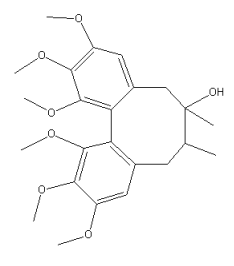

Schisandrin (5)

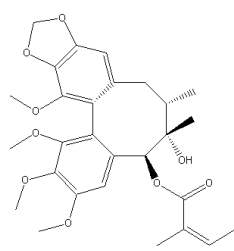

Schizantherin B (10)

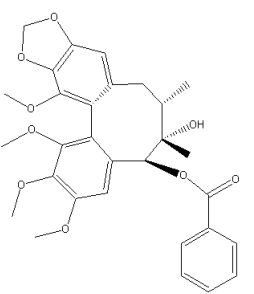

Schisantherin (15)

Figure 6. Chemical structures of 15 reference substances.

\subsection{Preparation of Standard Solutions}

A mixed standard stock solution containing 15 reference standards was prepared in methanol and their concentrations were as follows: $1,26.60 \mu \mathrm{g} / \mathrm{mL} ; 2,0.10 \mu \mathrm{g} / \mathrm{mL} ; 3,29.52 \mu \mathrm{g} / \mathrm{mL} ; 4,12.77 \mu \mathrm{g} / \mathrm{mL}$; 5, $9.31 \mu \mathrm{g} / \mathrm{mL} ; 6,1.01 \mu \mathrm{g} / \mathrm{mL} ; 7,0.93 \mu \mathrm{g} / \mathrm{mL} ; 8,5.35 \mu \mathrm{g} / \mathrm{mL} ; \mathbf{9}, 2.26 \mu \mathrm{g} / \mathrm{mL} ; 10,2.21 \mu \mathrm{g} / \mathrm{mL} ; \mathbf{1 1}$, $1.20 \mu \mathrm{g} / \mathrm{mL} ; \mathbf{1 2}, 2.40 \mu \mathrm{g} / \mathrm{mL} ; \mathbf{1 3}, 4.60 \mu \mathrm{g} / \mathrm{mL} ; \mathbf{1 4}, 1.47 \mu \mathrm{g} / \mathrm{mL} ; \mathbf{1 5}, 7.17 \mu \mathrm{g} / \mathrm{mL}$. This standard stock solution was then diluted with methanol to a series of appropriate concentrations to generate the calibration curves. The solutions were stored at $4{ }^{\circ} \mathrm{C}$ for a day prior to LC-MS analysis. 


\subsection{Preparation of Sample Solutions}

The dried fruits were pulverized into powders and screened through the 50-mesh sieve. Each sample $(0.5 \mathrm{~g})$ was accurately weighed and extracted by ultrasonication $(500 \mathrm{~W}, 40 \mathrm{kHz})$ in $20 \mathrm{~mL}$ methanol for $40 \mathrm{~min}$. After cooling down at room temperature, methanol was added to compensate for the weight lost during extraction. After centrifugation $(12,000 \mathrm{rpm}, 10 \mathrm{~min})$ and filtering $\left(0.22 \mu \mathrm{m}\right.$ membrane filter), the supernatants were stored in a sample plate at $4{ }^{\circ} \mathrm{C}$ prior to LC-MS analysis.

\subsection{Chromatographic and Mass Spectrometric Conditions}

The mass spectrometry detection was performed using an API5500 triple quadrupole mass (AB SCIEX, Framingham, MA, USA). The MS was equipped with an electrospray ionization (ESI) source operating in MRM and under both positive and negative ion modes. The MS parameters were set as follows: gas temperature $550{ }^{\circ} \mathrm{C}$; pressures of nebulizer of MS, $5500 \mathrm{~V}$ (positive) and $-4500 \mathrm{~V}$ (negative); GSI flow $65 \mathrm{~L} / \mathrm{min}$; CUR flow $30 \mathrm{~L} / \mathrm{min}$ and all MS data were acquired and analyzed using the Analyst 1.5.2 software (AB SCIEX, Framingham, MA, USA). The cone voltage and collision energy parameter of each compound were individually optimized.

The chromatographic analysis was performed on a Shimadzu SIL-20A XR system (Shimadzu, Kyoto, Japan), consisting of a binary solvent delivery system and an automatic sampler. A Synergi ${ }^{\mathrm{TM}}$ Hydro-RP $100 \AA ̊$ column $(100 \mathrm{~mm} \times 2.0 \mathrm{~mm}, 2.5 \mu \mathrm{m}$, (Phenomenex, Los Angeles, CA, USA) was used for eluting samples. The mobile phase was composed of water (A) and acetonitrile (B) using a gradient elution of $30 \%-52 \%$ B at $0-4 \mathrm{~min}, 52 \%-75 \%$ B at $4-8 \mathrm{~min}, 75 \%-90 \%$ B at $8-11 \mathrm{~min}, 90 \%-30 \%$ $\mathrm{B}$ at $11-15 \mathrm{~min}, 30 \% \mathrm{~B}$ at $15-17.10 \mathrm{~min}$. The column temperature was $40{ }^{\circ} \mathrm{C}$, the flow rate kept at $0.4 \mathrm{~mL} / \mathrm{min}$, and the sample injection volume was $1 \mu \mathrm{L}$.

\subsection{Validation of the Method}

\subsubsection{Linearity, LOD, and LOQ}

The linearity of the calibration curves was obtained by plotting the peak areas (Y) against the corresponding concentrations $(\mathrm{X})$ of each analyte. The lowest concentration of standard solution for calibration use was diluted with methanol to a series of appropriate concentrations. The LODs and LOQs of 15 analytes were determined using a series of diluted standard solutions until the signal-to-noise $(\mathrm{S} / \mathrm{N})$ ratios were about 3 and 10 , respectively.

\subsubsection{Precision, Repeatability, Stability, Accuracy}

The analysis method developed in this study was validated for precision (the intra- and inter-day), repeatability, stability, and accuracy. The intra-day and inter-day variability tests were determined by measuring the mixed standard solutions in six replicates in a day and once a day during three consecutive days, respectively. To evaluate the repeatability, six different analytical sample solutions prepared from the same sample (sample 1) were parallel processed and analyzed. To confirm the stability, the sample solution mentioned above was stored at room temperature and analyzed at 0 , $2,4,8,12$, and $24 \mathrm{~h}$, respectively. All the variations were expressed in RSD. A recovery test was used to evaluate the accuracy of this method. A certain amount of the 15 standards with low $(80 \%)$, medium (100\%), and high (120\%) levels were added into a known amount of samples $(0.25 \mathrm{~g})$, and then extracted and analyzed with the same procedures. To be specific, a recovery test was conducted by standard protocol and calculated by the formula: $(\%)=$ (found amount- original amount in sample)/spiked amount $\times 100 \%$. 


\subsection{Multivarite Statistical Analysis}

Multivariate statistical analysis was performed using the Simca-P 13.0 software (for Windows, Umetrics AB, Umeå, Sweden) by PCA and PLS-DA. PCA and PLS-DA were used to evaluate the variations of the two types of wuweizi according to the contents of the 15 components. PCA is an unsupervised pattern recognition method used for analyzing, classifying, and reducing the dimensionality of numerical datasets in a multivariate problem [20,21], and it has been widely used for the quality control of herbal medicines [22-24]. PLS-DA is good for highlighting the differences between two groups. It is possible to identify and select the important markers in samples via multivariate analysis of LC/MS data, even at low concentration levels [25]. Data of the contents of 15 compounds in wild and cultivated samples were listed. When the contents of investigated components were below the quantitation limit or not detected in the samples, the values of such elements were considered to be 0 . All experimental data were statistically analyzed by independent sample t-test (SPSS 16.0 for Windows, IBM, Armonk, NY, USA). The columns were charted by Origin pro 8 (Origin Lab, Northampton, MA, USA), showing the difference of each compound between two types of wuweizi. GRA provides a reliable guarantee for the quality evaluation of traditional Chinese medicine on the basis of the contents of the 15 index constituents [26].

\section{Conclusions}

An analytical method based on UFLC-QTRAP-MS/MS was used for the simultaneous determination of 15 components, including 11 lignans and 4 organic acids, in wuweizi under different ecosystems (wild and cultivated). Multivariate statistical analyses, such as PCA, PLS-DA, independent sample t-test, and GRA, have been successfully applied to comprehensively analyze and evaluate the wuweizi under different ecosystems according to the contents of the 15 components. The data of content determination showed that lignans had higher contents in wild-type, while cultivated-type contained more organic acids. This phenomenon is probably ascribed to environmental stress (such as water, soil strength, and nutrient status), which can have a great influence on the accumulation of the active compounds of medicinal plants. PCA and PLS-DA results showed that there are great difference between wild and cultivated wuweizi, and 4 different compounds (protocatechuic acid, quinic acid and L-(-)-malic acid, and schisandrol B) were significantly related to sample classification. It could be seen that the quality of the samples collected from the wild environment was better according to the GRA results. Wild wuweizi usually suffered more stress than cultivated-type and produced more specialized metabolites, even though the quality indicator of the two types all conformed to the requirements of Chinese pharmacopoeia. Findings from this research may provide a new method for the comprehensive evaluation and quality control of wuweizi from different ecosystems. It may also provide a basis for differentiating wild and cultivated wuweizi at the chemistry level.

Supplementary Materials: The following are available online. Table S1: Levels and factors of orthogonal table. Table S2: Result of $\mathrm{L}_{9}(3)^{3}$ orthogonal experiment. Table S3: Recoveries and relative standard deviations (RSD) of fifteen components $(\%, n=3)$. Table S4: Quality sequencing of the samples. Figure S1: Total ion chromatogram of the representative wild and cultivated samples.

Author Contributions: S.C. and J.S. carried out the experiments, analyzed data, and composed the draft of the manuscript. C.W., M.T., and J.C. proposed the study and helped to perform the experiments. L.Z., R.T., and J.M. provided the samples of wuweizi. X.L. participated in the supervision of this study and edited the manuscript. All authors read and approved the manuscript.

Funding: This research was supported by the Standardization of Chinese Materia Medica Program (No. ZYBZH-C-JS-32).

Conflicts of Interest: The authors declare no conflict of interest. 


\section{References}

1. Zhou, Y.; Huang, S.X.; Pu, J.X.; Li, J.R.; Ding, L.S.; Chen, D.F.; Sun, H.D.; Xu, H.X. Ultra performance liquid chromatography coupled with quadrupole time-of-flight mass spectrometric procedure for qualitative and quantitative analyses of nortriterpenoids and lignans in the genus Schisandra. J. Pharmaceut. Biomed. 2011, 56, 916-927. [CrossRef]

2. Szopa, A.; Ekiert, R.; Ekiert, H. Current knowledge of Schisandra chinensis (Turcz.) Baill. (Chinese magnolia vine) as a medicinal plant species: A review on the bioactive components, pharmacological properties, analytical and biotechnological studies. Phytochem. Rev. 2017, 16, 195-215. [CrossRef]

3. Li, X.G.; Gao, Q.; Weng, W.; Zhang, P.F.; Xiao, F.; Luo, H.M. Research progress of effective parts and its pharmacological action of Schisandrae Chinesis Fructus. J. Chin. Med. Mater. 2005, 28, 156-159.

4. Hu, J.Y.; Mao, C.Q.; Gong, X.D.; Lu, T.L.; Chen, H.; Huang, Z.J.; Cai, B.C. Simultaneous determination of eleven characteristic lignans in Schisandra chinensis by high-performance liquid chromatography. Pharmacogn. Mag. 2013, 9, 155-161.

5. Ming, Y.B.; Zhao, H.; Zhou, L.; Tian, Y.H. Reseach progress of Schisandra Chinesis (Turcz) Bail. Pract. Pharm. Clin. Remed. 2007, 10, 365-367.

6. Kormosh, N.; Laktionov, K.; Antoshechkina, M. Effect of a combination of extracts from several plants on cell-mediated and humoral immunity of patients with advanced ovarian cancer. Phytother. Res. 2006, 20, 424-425. [CrossRef]

7. Liu, K.T.; Cresteil, T.; Columelli, S.; Lesca, P. Pharmacological properties of dibenzo[a,c]cyclooctene derivatives isolated from Fsh, N.; LaII. Induction of phenobarbital-like hepatic monooxygenases. Chem-Biol. Interact. 1982, 39, 315-330. [CrossRef]

8. Yang, H.J.; Yang, S.M. General situation of pharmacological action of Fructus Schisandra Chinesis (Turcz) Bail. Ginseng Res. 1999, 11, 5-8.

9. Zhong, S.; Nie, Y.C.; Gan, Z.Y.; Liu, X.D.; Fang, Z.F.; Zhong, B.N.; Tian, J.; Huang, C.Q.; Lai, K.F.; Zhong, N.S. Effects of Schisandra chinensis extracts on cough and pulmonary inflammation in a cough hypersensitivity guinea pig model induced by cigarette smoke exposure. J. Ethnopharmacol. 2015, 165, 73-82. [CrossRef]

10. Wang, C.C.; Cai, H.; Zhao, H.; Yan, Y.; Shi, J.J.; Chen, S.Y.; Tan, M.X.; Chen, J.L.; Zou, L.S.; Chen, C.H. Distribution patterns for metabolites in medicinal parts of wild and cultivated licorice. J. Pharm. Biomed. Anal. 2018, 161, 464-473. [CrossRef]

11. Brunetti, C.; George, R.M.; Tattini, M.; Field, K.; Davey, M.P. Metabolomics in plant environmental physiology. J. Exp. Bot. 2013, 64, 4011-4020. [CrossRef] [PubMed]

12. Astaf'eva, O.V.; Sukhenko, L.T. Comparative analysis of antibacterial properties and chemical composition of Glycyrrhiza glabra L. from Astrakhan region (Russia) and Calabria region (Italy). Bull. Exp. Biol. Med. 2014, 156, 829-832. [CrossRef]

13. Wei, S.S.; Yang, M.; Chen, X.; Wang, Q.R.; Cui, Y.J. Simultaneous determination and assignment of 13 major flavonoids and glycyrrhizic acid in licorices by HPLC-DAD and Orbirap mass spectrometry analyses. Chin. J. Nat. Med. 2015, 13, 232-240. [CrossRef]

14. Ren, M.T.; Chen, J.; Song, Y.; Sheng, L.S.; Li, P.; Qi, L.W. Identification and quantification of 32 bioactive compounds in Lonicera species by high performance liquid chromatography coupled with time-of-flight mass spectrometry. J. Pharm. Biomed. Anal. 2008, 48, 1351-1360. [CrossRef] [PubMed]

15. Deng, X.X.; Chen, X.H.; Cheng, W.M.; Cheng, W.M.; Shen, Z.D.; Bi, K.S. Simultaneous LC-MS Quantification of 15 Lignans in Schisandra chinensis (Turcz.) Baill. Fruit. Chromatographia 2008, 67, 559-566. [CrossRef]

16. Zhang, H.; Zhang, G.Q.; Zhu, Z.Y.; Zhao, L.; Fei, Y.; Jing, J.; Chai, Y.F. Determination of six lignans in Schisandra chinensis (Turcz.) Baill. Fruits and related Chinese multiherb remedies by HPLC. Food Chem. 2009, 115, 735-739. [CrossRef]

17. Liu, H.; Lai, H.; Jia, X.; Liu, J.; Zhang, Z.; Qi, Y.; Zhang, J.; Song, J.; Wu, C.; Zhang, B. Comprehensive chemical analysis of Schisandra chinensis by HPLC-DAD-MS combined with chemometrics. Phytomedicine. 2013, 20, 1135-1143. [CrossRef]

18. An, K.L.; Li, D.K.; Zhou, D.Z.; Ye, Z.L.; Guo, Q.S. Effects of different drying methods on quality of schisandrae chinensis fructus. Chin. J. Chin. Mater. Med. 2014, 39, 2900-2906.

19. Xu, J.H.; Su, L.L.; Wang, Q.H.; Mao, C.Q.; Wang, D.D. Determination of citric acid, L-malic acid and 5-hydroxyl methyl furfural in diffe-rent processed products of Schisandra chinensis by HPLC. Northwest Pharm. J. 2017, 32, 548-551. 
20. Ji-ye, A.; He, J.; Sun, R.B. Multivariate statistical analysis for metabolomic data: the key points in principal component analysis. Acta Pharm. Sin. 2018, 53, 929-937.

21. Bo, N.; Broberg, P.; Lindberg, C.; Plymoth, A. Analysis and understanding of high-dimensionality data by means of multivariate data analysis. Chem. Bio-divers. 2005, 2, 1487-1494.

22. Mediani, A.; Abas, F.; Maulidiani, M.; Khatib, A.; Tan, C.P.; Ismail, I.S.; Shaari, K.; Ismail, A. Characterization of Metabolite Profile in Phyllanthus niruri and Correlation with Bioactivity Elucidated by Nuclear Magnetic Resonance Based Metabolomics. Molecules 2017, 22, 902. [CrossRef]

23. Han, D.Q.; Zhao, J.; Xu, J.; Peng, H.S.; Chen, X.J.; Li, S.P. Quality evaluation of Polygonum multiflorum in China based on HPLC analysis of hydrophilic bioactive compounds and chemometrics. J. Pharm. Biomed. Anal. 2013, 72, 223-230. [CrossRef] [PubMed]

24. Da, J.; Wu, W.Y.; Hou, J.J.; Long, H.L.; Yao, S.; Yang, Z.; Cai, L.Y.; Yang, M.; Jiang, B.H.; Liu, X.; et al. Comparison of two officinal Chinese pharmacopoeia species of Ganoderma based on chemical research with multiple technologies and chemometrics analysis. J. Chromatogr. A. 2012, 1222, 59-70. [CrossRef] [PubMed]

25. Xu, X.; Xu, S.; Zhang, Y.; Zhang, H.; Liu, M.N.; Liu, H.; Gao, Y.; Xue, X.; Xiong, H.; Lin, R.; et al. Chemical Comparison of Two Drying Methods of Mountain Cultivated Ginseng by UPLC-QTOF-MS/MS and Multivariate Statistical Analysis. Molecules 2017, 22, 717. [CrossRef] [PubMed]

26. Chen, C.H.; Liu, Z.X.; Zou, L.S.; Liu, X.H.; Chai, C.; Zhao, H.; Yan, Y.; Wang, C.C. Quality evaluation of Apocyni Veneti Folium from different habitats and commercial herbs based on simultaneous determination of multiple bioactive constituents combined with multivariate statistical analysis. Molecules 2018, 23, 573. [CrossRef] [PubMed]

Sample Availability: Samples of the compounds are available from the authors.

(C) 2019 by the authors. Licensee MDPI, Basel, Switzerland. This article is an open access article distributed under the terms and conditions of the Creative Commons Attribution (CC BY) license (http://creativecommons.org/licenses/by/4.0/). 